Pre-print to Letter with ChemistryWorld, Magazine of the Royal Society of Chemistry. Link: https://protect-eu.mimecast.com/s/2-mKCL7VJCRjK2YSPhQnO?domain=chemistryworld.com Published in print in the March 2021 issue.

\title{
Do We Need Affirmative Action?
}

\section{8 words}

Diversity is crucial to excellence in innovation and discovery for the good of humanity and fundamental knowledge; in the chemical sciences and beyond. Yet, data show that progress in diversity, equity, and inclusion (DEI) has proceeded at a glacial pace. This is in spite of the UK's 1970s-1990s equality and antidiscrimination laws, and their broadening in 2010 under the Equality Act. Legislation intended to spur rapid progress in DEl in every sector and at every level of employ. Our community trajectory is not as it should be - the Equality Act and voluntary schemes such as Disability Confident are thus proven grossly underwhelming. What more is needed?

During the Royal Society of Chemistry's (RSC) online Inclusion and Diversity Forum in December a participant stated that affirmative action is not legal during hiring in the UK. Having worked in the US where affirmative action is generally a business-as-usual approach to staff recruitment and diversification my immediate response was 'change the law'. Is such reformist thinking widespread? Why oh why does the UK allow positive action but not permit affirmative action? Surely we should have the possibility to set firm policies and practices with target quotas so as to more rapidly uplift groups of people that are currently minoritised and marginalised. Are the legality of such actionable levers key to accelerating culture change and the shattering of persistent barriers that thwart progress in DEI? Like many of you I'm no legal eagle or trade union leader. Given BREXIT and the ongoing work of the All-Party Parliamentary Group on Diversity and Inclusion in STEM, is the UK reviewing its employment laws? Is now a prime time to speak out and insist on affirmative action for the betterment of society and science?

By Amy J. V. Riches

Planetary Scientist, Geochemist, Author and Editor

Visiting Honorary Fellow, University of Edinburgh, UK, and Affiliate Scientist, SETI Institute, USA.

Website: https://amyriches.org/ 